\title{
Synergistic Fungicidal Activities of Amphotericin B and N-Methyl-N"-dodecylguanidine: A Constituent of Polyol Macrolide Antibiotic Niphimycin
}

\author{
Akira Ogita, Keiji Matsumoto, Ken-ichi Fujita, Yoshinosuke Usuki, Yoshiro Hatanaka, \\ Toshio Tanaka
}

Received: November 9, 2006 / Accepted: December 20, 2006

(C) Japan Antibiotics Research Association

\begin{abstract}
The synergy between the alkylguanidinium chain of niphimycin (NM), a polyol macrolide antibiotic, and polyene macrolide amphotericin $\mathrm{B}(\mathrm{AmB})$ without such an alkyl side chain was examined using $N$-methyl- $N^{\prime \prime}$ alkylguanidines as its synthetic analogs. Among the analogs, $N$-methyl- $N^{\prime \prime}$-dodecylguanidine (MC12) most strongly inhibited the growth of Saccharomyces cerevisiae cells and those of other fungal strains in synergy with AmB. MC12 itself was not lethal but the analog could be a cause of a rapid cell death progression of yeast cells in the presence of $\mathrm{AmB}$ at a nonlethal concentration. Their combined actions resulted in the generation of NM-like fungicidal activity that depended on plasma membrane disability and cellular reactive oxygen species production. We also found an aberrant vacuolar morphogenesis and an associated vacuolar membrane disability in cells treated simultaneously with MC12 and $\mathrm{AmB}$, as in the case of NM-treated cells. These findings support the idea that the alkylguanidinium chain plays a major role in the fungicidal activity of NM in cooperation with the polyol lactone ring as its enhancer.
\end{abstract}

Keywords niphimycin, amphotericin B, synergy, vacuole, Saccharomyces cerevisiae

T. Tanaka (Corresponding author), K. Matsumoto, K. Fujita, Y. Usuki: Graduate School of Science, Osaka City University, 3-3-138 Sugimoto, Sumiyoshi-ku, Osaka 558-8585, Japan, E-mail: tanakato@sci.osaka-cu.ac.jp

\section{Introduction}

Niphimycin (NM, Fig. 1) was first isolated from a strain of Streptomyces hygroscopicus, and was designated a guanidylpolyol macrolide antibiotic because of its structure consisting of polyol lactone ring and an alkylguanidinium chain $[1,2]$. Despite some structural analogy to amphotericin B (AmB, Fig. 1), a polyene macrolide antibiotic, NM shows broad antimicrobial activities against Gram-positive bacteria, which are absolutely resistant to $\mathrm{AmB}$, in addition to filamentous fungi and yeasts [3]. The mode of fungicidal activity of $\mathrm{NM}$ is apparently distinguishable from that of $\mathrm{AmB}$ as observed from the disruptive effect of NM on the yeast plasma membrane by its direct interaction with phospholipids, but not with ergosterol, a molecular target of AmB [4]. We also found the involvement of cellular reactive oxygen species (ROS), which are not generated in AmB-treated cells, in the fungicidal activity of NM.

Our attention was thus focused on the role of the alkylguanidinium chain of NM, which is absent from the structure of AmB, in the fungicidal activity of NM. Among several synthetic analogs of the alkylguanidinium chain, $N$ methyl- $N$ "-hexadecylguanidine (MC16, Fig. 1) was found to exert the highest fungicidal activity by directly damaging
A. Ogita: Research Center for Urban Health and Sports, Osaka City University, 3-3-138 Sugimoto, Sumiyoshi-ku, Osaka 5588585, Japan

Y. Hatanaka: Osaka Municipal Technical Research Institute, 1-650 Morinomiya, Joto-ku, Osaka 536-8553, Japan 

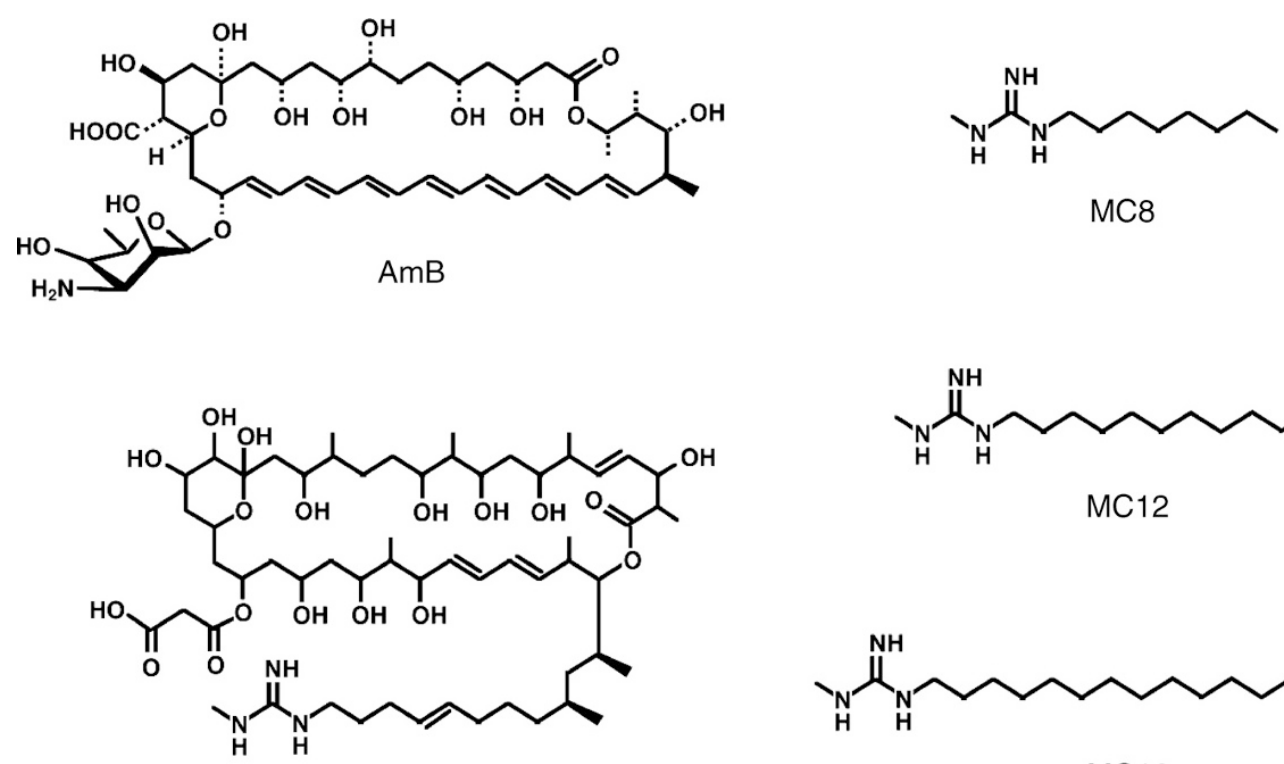

NM

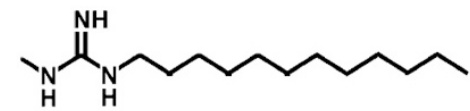

$\mathrm{MC} 12$

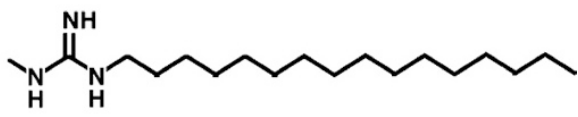

MC16

Fig. 1 Structures of amphotericin B (AmB), niphimycin (NM), N-methyl- $N^{\prime \prime}$-octylguanidine (MC8), $N$-methyl- $N$ "dodecylguanidine (MC12), and $N$-methyl- $N$ "-hexadecylguanidine(MC16).

the plasma membrane and inducing ROS generation in a NM-like manner [5]. However, MIC of MC16 was much higher than that of NM, suggesting the role of the polyol lactone ring as a constituent that enhances the interaction of the alkylguanidinium chain with a corresponding target molecule. The polyol lactone ring of NM may alternatively exhibit its own fungicidal activity as expected from the polyene lactone ring of AmB without such an alkyl side chain. This constituent has never been prepared by the chemical degradation of NM or by any other methods and thus its physiological function remains unclear.

We therefore examined the effects of the simultaneous application of the alkylguanidine and $\mathrm{AmB}$ as a substitute for the polyol lactone ring of NM. It was also valuable to confirm whether AmB can enhance the antifungal activity other than that dependent on the plasma membrane permeability change. $N$-Methyl- $N N^{\prime \prime}$-dodecylguanidine (MC12) was not lethal but this analog could strongly inhibit the growth of Saccharomyces cerevisiae and some other fungal strains when used in combination with AmB. Their combined actions resulted in the generation of NM-like fungicidal activity that depended on plasma membrane disability and cellular ROS production in S. cerevisiae cells. In addition, we found an aberrant vacuole morphogenesis in cells treated with $\mathrm{MC} 12$ and $\mathrm{AmB}$ each at the nonlethal concentration, as in the case with NMtreated cells.

\section{Materials and Methods}

\section{Measurement of Cell Growth and Viability}

MICs of $N$-methyl- $N N^{\prime \prime}$-alkylguanidines, fatty acids, and alkylamines were determined for S. cerevisiae X 2180-1A (a) in the presence or absence of $\mathrm{AmB}$ by the serial 2-fold broth dilution method as follows [6]. After diluting an overnight culture with YPD medium (yeast extract $1 \%$, peptone $2 \%$, and glucose $2 \%$ ) to $10^{6}$ cells $/ \mathrm{ml}$, the cell suspension was incubated in a freshly prepared medium in a 96-well microplate at $30^{\circ} \mathrm{C}$ for 48 hours. MICs of MC12 and $\mathrm{AmB}$ were also determined for Candida albicans IFO1061, Aspergillus niger ATCC 6275, Rhizopus oryzae IFO 4766 and Bacillus subtilis IFO 3007 in addition to $S$. cerevisiae X2180-1A (a) by applying the checkerboard technique [7]. The filamentous fungi were precultivated in $2.5 \%$ malt extract medium (Oriental Yeast Co., Tokyo, Japan) at $30^{\circ} \mathrm{C}$ for 24 hours and the overnight cultures were diluted 100 -fold with the same medium for MIC determination at $30^{\circ} \mathrm{C}$ for 48 hours.

S. cerevisiae $\mathrm{X} 2180-1 \mathrm{~A}$ (a) was used in the following experiments to examine the effects of $\mathrm{MC} 12, \mathrm{AmB}$ and their combination on the growth and other properties of the yeast cells. Cells from an overnight culture were incubated in YPD medium at an initial cell density of approximately $10^{7}$ cells $/ \mathrm{ml}$ in the presence or absence of each compound at $30^{\circ} \mathrm{C}$ for the indicated times. The cell viability was 
determined by counting viable cell number as colony forming units [8].

\section{Assay of Plasma Membrane Permeability Change}

The plasma membrane permeability change was assayed by measuring the efflux of cytoplasmic $\mathrm{K}^{+}$as follows. Overnight cultured cells were harvested by centrifugation, washed with $50 \mathrm{mM}$ succinate buffer $(\mathrm{pH}$ 6.0) and suspended in the buffer to obtain a density of $10^{8}$ cells $/ \mathrm{ml}$. The cell suspension with or without the addition of each compound was shaken at $30^{\circ} \mathrm{C}$ and the supernatant obtained after removing cells by centrifugation was assayed for its content of $\mathrm{K}^{+}$according to our previously described method [4].

\section{Assay of Cellular ROS Production}

The effects of MC12, AmB and their combination on cellular ROS productions were assayed by following a previously described method [9]. Cells from an overnight culture were inoculated into a freshly prepared medium to obtain a density of approximately $10^{7}$ cells $/ \mathrm{ml}$. Cells were then incubated with $40 \mu \mathrm{M} \mathrm{2} 2^{\prime}, 7^{\prime}$-dichlorodihydrofluorescein diacetate at $30^{\circ} \mathrm{C}$ for 60 minutes, and then collected by centrifugation and suspended in an equal volume of the medium. The cell suspensions $(1.0 \mathrm{ml})$ were further treated with or without each compound for 60 minutes, and then washed and resuspended in $100 \mu \mathrm{l}$ of phosphate-buffered saline. Fluorescence from cells was then measured with excitation at $480 \mathrm{~nm}$ and emission at $530 \mathrm{~nm}$.

\section{Vacuole Staining}

Vacuoles were stained with a fluorescent probe, FM4-64 ( $N$-(3-triethylammoniumpropyl)-4-(6-(4-(diethylamino)phenyl)hexatrienyl)pyridinium dibromide) as follows [10]. Cells from an overnight culture were inoculated into a freshly prepared medium to obtain a density of approximately $10^{7}$ cells $/ \mathrm{ml}$. After incubating with $5 \mu \mathrm{M}$ FM4 -64 at $30^{\circ} \mathrm{C}$ for 30 minutes, cells were collected by centrifugation, suspended in a fresh medium, and incubated again in the presence or absence of each compound at $30^{\circ} \mathrm{C}$ for 30 minutes. Cells were then observed under a phase-contrast microscope and a fluorescence microscope with excitation at $480 \mathrm{~nm}$ and emission at $530 \mathrm{~nm}$.

\section{Transmission Electron Microscopy}

Cells were treated for transmission electron microscopy as follows. An overnight grown culture was inoculated into freshly prepared medium to obtain an initial cell density of approximately $10^{7} \mathrm{cell} / \mathrm{s} / \mathrm{ml}$. Cells were then grown in the presence or absence of each compound $30^{\circ} \mathrm{C}$ for 60 minutes with vigorous shaking. After fixing with $2.5 \%$ (v/v) glutaraldehyde at room temperature for 3 hours, cells were washed three times in $0.1 \mathrm{M}$ sodium potassium phosphate buffer $(\mathrm{pH} 7.0)$ and postfixed with $2 \%(\mathrm{v} / \mathrm{v})$ osmium tetroxide for 60 minutes. Cells were then dehydrated with 10 -minutes serial ethanol washes $(50,70$, 90,95 , and 4 times in $100 \%$ ) and infiltrated with $25 \%$ quetol 651 in $75 \%$ ethanol on a rotator overnight at room temperature. The ratio of quetol was increased gradually to $100 \%$ for 3 days. Cells were pelleted, transferred to capsules, overlaid with fresh quetol for polymerization at $65^{\circ} \mathrm{C}$ for $24 \sim 48$ hours. Thin sections were cut, stained with uranyl acetate and lead acetate, and observed under a transmission electron microscope.

\section{Chemicals}

$N$-Methyl- $N "$-octylguanidine (MC8), MC12, and MC16 were synthesized as described previously [5]. FM4-64 was a product of Molecular Probes (Eugene, OR, USA). Other chemicals were obtained from Sigma Chemical Co. (St. Louis, MO, USA) unless otherwise stated.

\section{Results and Discussion}

\section{Synergy between MC12 and AmB}

We first measured MICs of MC8, MC12 and MC16 (Fig. 1) against $S$. cerevisiae in the presence or absence of $0.5 \mu \mathrm{M}$ $\mathrm{AmB}$ in comparison with the compounds having long-chain alkyl moieties such as free fatty acids and alkylamines. $\mathrm{AmB}$ did not inhibit yeast cell growth at this concentration of $0.5 \mu \mathrm{M}$ as its MIC was determined to be $1 \mu \mathrm{M}$. As summarized in Table 1 , free fatty acids tested did not

Table 1 MICs of fatty acids, alkylamines, and $\mathrm{N}$-methyl$N^{\prime \prime}$-alkyl guanidine derivates in the presence or absence of AmB against Saccharomyces cerevisiae cells

\begin{tabular}{lrc}
\hline & \multicolumn{2}{c}{ MIC $(\mu \mathrm{M})$ with } \\
\cline { 2 - 3 } Test compounds & None & AmB $^{\mathrm{a}}$ \\
\cline { 2 - 3 } & $>400$ & $>400$ \\
Lauric acid & $>400$ & $>400$ \\
Myristic acid & $>400$ & $>400$ \\
Palmitic acid & $>400$ & $>400$ \\
Octylamine & 50 & 25 \\
Laurylamine & 50 & 25 \\
Hexadecylamine & $>400$ & $>400$ \\
MC8 & 100 & 12.5 \\
MC12 & 100 & \\
MC16 & & \\
\hline
\end{tabular}

${ }^{a}$ Amphotericin B (AmB) was added at $0.5 \mu \mathrm{M}$. 
inhibit the growth of S. cerevisiae cells at $400 \mu \mathrm{M}$ even in the presence of AmB regardless of the length of their alkyl or acyl chains. Alkylamine with the lauryl or hexadecyl chain, but not with the octyl chain, inhibited the yeast cell growth at $50 \mu \mathrm{M}$. However, their MICs only slightly decreased in the presence of $\mathrm{AmB}$, reflecting the lack of effective synergy between the actions of alkylamine and this polyene macrolide antibiotic. MC8 showed no growth inhibitory activity with or without AmB. In contrast, the presence of AmB could significantly decrease the MICs of MC12 and MC16, which were commonly detected at $100 \mu \mathrm{M}$ in the absence of $0.5 \mu \mathrm{M} \mathrm{AmB}$. Our previous study suggests that the polyol lactone ring of NM functions as a carrier of the alkylguanidinium chain which enables the interaction of the chain with the corresponding molecular target [5]. This study rather supported the idea that the polyol lactone ring of NM can potentiate the fungicidal activity of the alkylguanidinium chain via the AmB-like effect even if the chain is not covalently bound to the ring. Such a potentiation of the antifungal activity of MC12 or MC16 could not be simply attributed to its enhanced incorporation into or across the plasma membrane due to AmB-induced plasma membrane permeability change, as seen from the failure of $\mathrm{AmB}$ in elevating the antifungal activity of alkylamine.
We next applied a checkerboard technique to evaluate whether $\mathrm{MC} 12$ and $\mathrm{AmB}$ can inhibit the growth of $S$. cerevisiae and other fungal strains in a synergistic manner. Typical isobolograms demonstrated the appearance of synergy between the actions of $\mathrm{MC} 12$ and $\mathrm{AmB}$ was greater against filamentous fungi such as $A$. niger and $R$. oryzae than against yeasts such as $S$. cerevisiae and $C$. albicans, as shown in Fig. 2. Although MC12 inhibits the growth of Gram-positive bacteria similarly to NM [3 5], its growth inhibitory activity against $B$. subtilis was scarcely enhanced in the presence of $\mathrm{AmB}$ (data not shown). These findings suggest that their combined actions fundamentally depend on the fungicidal activity of AmB, which has no molecular targets in bacterial cells.

In cultivation with shaking, MC12 decreased the growth rate of $S$. cerevisiae cells in a dose-dependent manner but caused no lethal damage even at the highest concentration (Fig. 3a) over the 4 hours incubation period. AmB was ineffective in inducing cell death at $1 \mu \mathrm{M}$, whereas this antibiotic could weakly decrease cell viability when its concentration was increased up to $2 \mu \mathrm{M}$ (Fig. $3 \mathrm{~b}$ ) over the 4 hours period. However, their combined actions resulted in more serious cell death progression even when $\mathrm{MC12}$ and AmB were added at the nonlethal concentrations of $50 \mu \mathrm{M}$ and $0.5 \mu \mathrm{M}$, respectively. The lethal damage much more

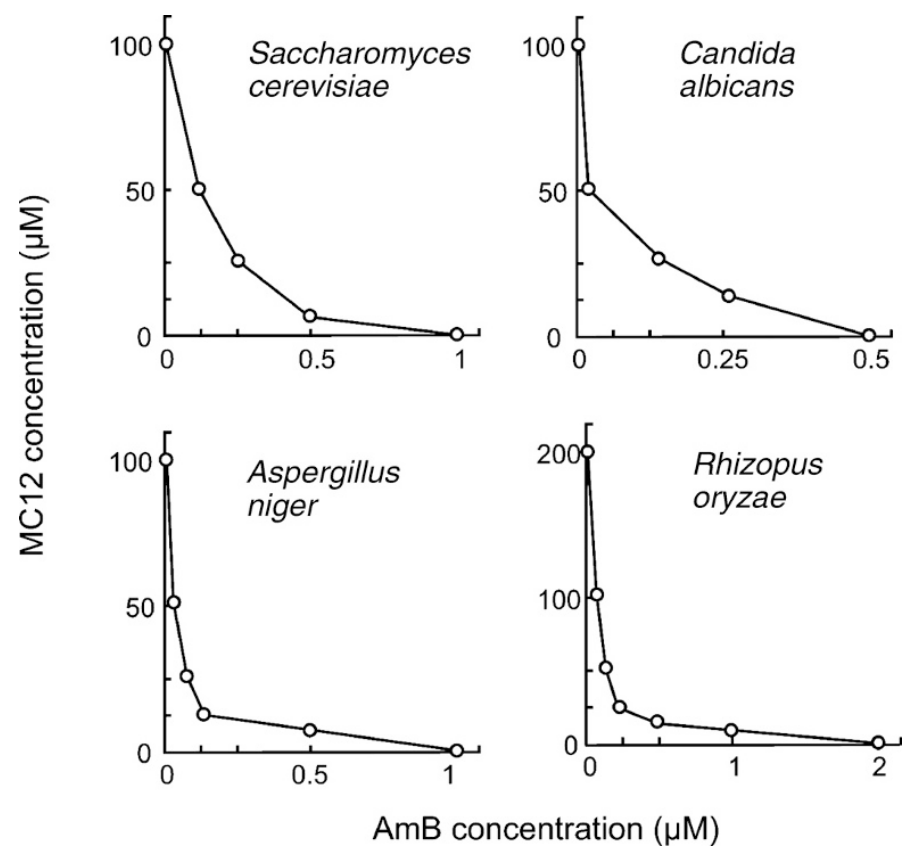

Fig. 2 Isobolograms demonstrating synergy of MC12 and AmB against Saccharomyces cerevisiae, Candida albicans, Aspergillus niger, and Rhizopus oryzae.

Each point represents a combination of both agents that is needed for minimum growth inhibitory activity. The concentrations of MC12 and $\mathrm{AmB}$ are expressed as the fraction of one MIC for each agent when used alone. An isobol that bows toward the coordinates indicates synergism, a straight line isobol indicates indifference, and an isobol that bows away from the coordinates indicates antagonism. 
rapidly appeared in the medium in which the concentration of $\mathrm{AmB}$ was increased up to $1 \mu \mathrm{M}$ (Fig. 3c).

\section{Effects of MC12, AmB and Their Combination on Plasma Membrane Permeability}

The fungicidal activity of AmB has been mainly elucidated on the basis of its promotive effect on the plasma membrane permeability change [11]. This antibiotic directly interacts with sterols constituting the plasma membrane of eukaryotic microbial cells and thus can cause a change in the permeability of fungal plasma membrane
[12]. We therefore examined the mode of synergy between MC12 and AmB on the basis of their effects on plasma membrane permeability to $\mathrm{K}^{+}$. As shown in Fig. 4, MC12 alone at $50 \mu \mathrm{M}$ or even at $100 \mu \mathrm{M}$ did not promote $\mathrm{K}^{+}$ efflux from cells, as deduced from the loss of cell death induction (Fig. 3). AmB was effective in inducing the leakage of $\mathrm{K}^{+}$from cells even at $0.5 \mu \mathrm{M}$, and the rate of AmB-induced $\mathrm{K}^{+}$efflux was slightly higher in the medium containing this antibiotic at $1 \mu \mathrm{M}$. However, cells characteristically showed an extremely high rate of $\mathrm{K}^{+}$ efflux at the initial step of cell death induced by the
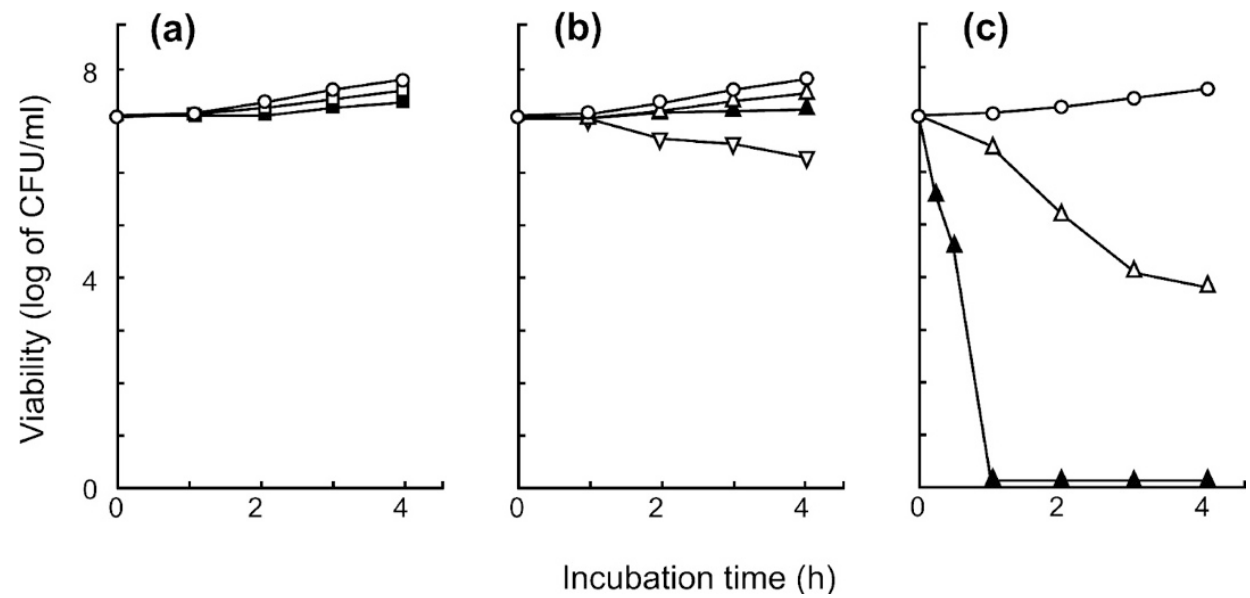

Fig. 3 Effects of MC12, AmB and combination of MC12 and AmB on growth of Saccharomyces cerevisiae cells.

For (a), cells $\left(10^{7} / \mathrm{ml}\right)$ were incubated in YPD medium containing MC12 at $0(0), 50(\square)$, and $100 \mu \mathrm{M}(\mathbf{\square})$. For (b), cells were incubated in YPD medium containing AmB at $0(O), 0.5(\triangle), 1(\mathbf{\Delta})$, and $2 \mu \mathrm{M}(\nabla)$. For $(\mathrm{c})$, cells were incubated in YPD medium containing $50 \mu \mathrm{M} \mathrm{MC} 12$ and $\mathrm{AmB}$ at $0(\bigcirc), 0.5(\triangle)$ and $1 \mu \mathrm{M}(\mathbf{\Delta})$.
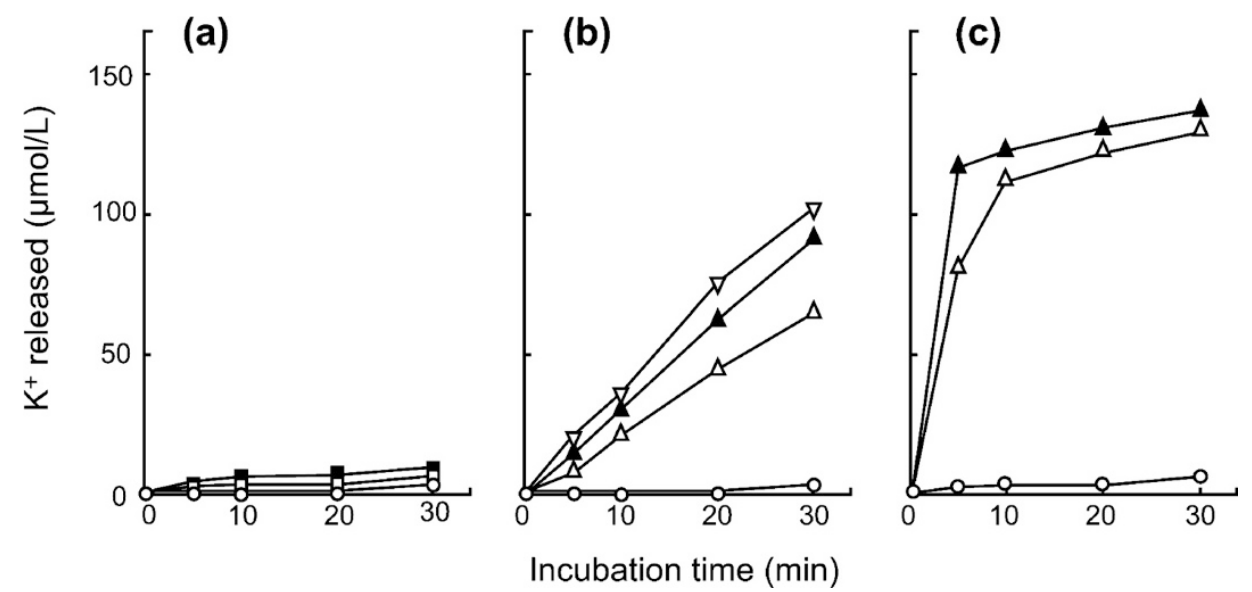

Fig. 4 Promotive effects of $\mathrm{MC12}, \mathrm{AmB}$, and combination of $\mathrm{MC12}$ and $\mathrm{AmB}$ on leakage of $\mathrm{K}^{+}$from Saccharomyces cerevisiae cells.

For (a), cells $\left(10^{8} / \mathrm{ml}\right)$ were incubated in $50 \mathrm{mM}$ succinate buffer $(\mathrm{pH} 6.0)$ containing MC12 at $0(O), 50$ ( $\square$ ), and $100 \mu \mathrm{M}(\mathbf{\square})$. For (b), cells $\left(10^{8} / \mathrm{ml}\right)$ were incubated in $50 \mathrm{mM}$ succinate buffer $(\mathrm{pH} 6.0)$ containing AmB at $0(O), 0.5(\triangle), 1(\mathbf{\Delta})$, and $2 \mu \mathrm{M}(\nabla)$. For (c), cells $\left(10^{8} / \mathrm{ml}\right)$ were incubated in $50 \mathrm{mM}$ succinate buffer (pH 6.0) containing $50 \mu \mathrm{M} \mathrm{MC12}$ and $\mathrm{AmB}$ at $0(\bigcirc), 0.5(\triangle)$, and $1 \mu \mathrm{M}(\mathbf{\Delta})$. 
combined actions of MC12 and $\mathrm{AmB}$ each at the nonlethal concentration. Such a marked change in plasma membrane permeability suggests that $\mathrm{MC} 12$ enhances the formation of a complex between $\mathrm{AmB}$ and ergosterol for $\mathrm{K}^{+}$channel formation across the plasma membrane.

\section{Effects of MC12, AmB and Their Combination on Cellular ROS Production}

The involvement of oxidative damage has been suggested for the fungicidal activity of $\mathrm{AmB}$ against $C$. albicans and its lytic activity on mammalian cells [13 15]. On the contrary, the antibiotic itself is evaluated as an antioxidant, and furthermore the antibiotic is able to exhibit a synergistic fungicidal activity against $C$. albicans when added with an antioxidant such as gallate [16 18]. We first applied a selective fluorescent probe for detection of ROS in $S$. cerevisiae cells in order to characterize the difference in the mode of fungicidal activity between $\mathrm{AmB}$ and NM, and found ROS predominant production only in NMtreated cells but not in AmB treated cells [4]. The alkylguanidinium chain was thus considered to be a constituent responsible for cellular ROS production and related plasma membrane disruptive damage. We likewise examined whether MC12 accelerated this cytotoxic event by its own action or in combination with AmB. As shown in Fig. 5, ROS production was promoted in the medium with MC12 to a significant extent and was markedly accelerated in cells treated simultaneous with MC12 and $\mathrm{AmB}$ each at the nonlethal concentration. This agrees with the role of $\mathrm{AmB}$ as an enhancer of MC12-dependent fungicidal activity.

\section{Effects of MC12, AmB and Their Combination on Vacuole Morphology and Function}

The vacuole is an organelle essential for plant and fungal cell function including osmotic adaptation [19]. nss 1 mutants of $S$. cerevisiae, which are characterized by severe vacuolar protein sorting and vacuolar morphological defects, are highly sensitive to polyene macrolide antibiotics [20]. On the basis of this finding, we have recently determined the yeast vacuole as a target of the fungicidal activity of $\mathrm{AmB}$ when cells are incubated under hypoosmotic

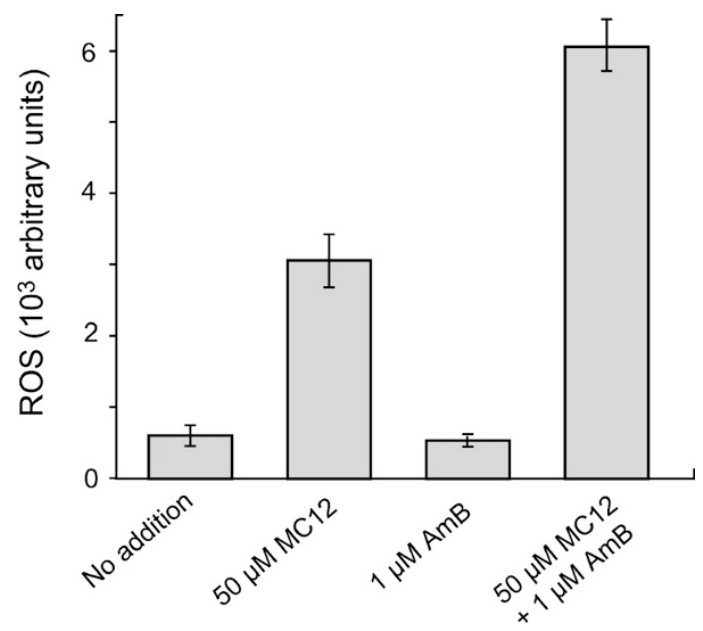

Fig. 5 Promotive effects of $\mathrm{MC12}, \mathrm{AmB}$, and combination of $\mathrm{MC12}$ and $\mathrm{AmB}$ on ROS production of Saccharomyces cerevisiae cells.

After DCFH-DA treatment, cells $\left(10^{7} / \mathrm{ml}\right)$ were incubated in YPD medium alone, the medium containing $50 \mu \mathrm{M} \mathrm{MC12,} \mathrm{the}$ medium containing $1 \mu \mathrm{M} \mathrm{AmB}$, and the medium containing both $50 \mu \mathrm{M} \mathrm{MC} 12$ and $1 \mu \mathrm{M} \mathrm{AmB}$ at $30^{\circ} \mathrm{C}$ for 60 minutes. (a)

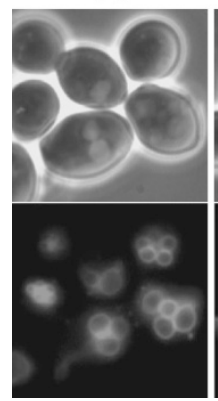

(b)

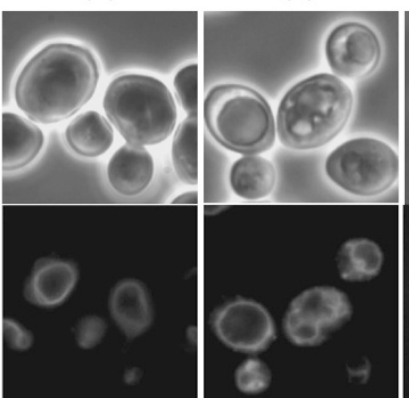

(d)

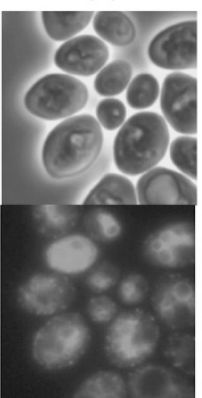

(e)

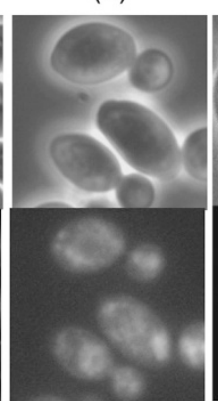

(f)

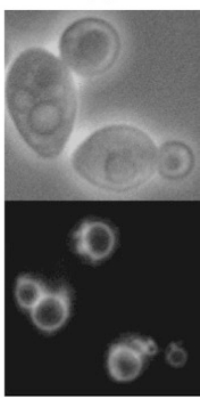

Fig. 6 Effects of $\mathrm{MC12}, \mathrm{AmB}, \mathrm{NM}$, and combination of $\mathrm{MC12}$ and $\mathrm{AmB}$ on vacuole morphology of Saccharomyces cerevisiae cells.

After FM4-64 fluorescent dye treatment, cells $\left(10^{7} / \mathrm{ml}\right)$ were incubated in YPD medium alone (a), the medium containing $50 \mu \mathrm{M}$ MC12 (b), the medium containing $1 \mu \mathrm{M} \mathrm{AmB}$ (c), the medium containing both $50 \mu \mathrm{M} \mathrm{MC12}$ and $1 \mu \mathrm{M}$ AmB (d), the medium containing $4 \mu \mathrm{M} \mathrm{NM}$ (e), and the medium containing $10 \mu \mathrm{M} \mathrm{AmB} \mathrm{(f)} \mathrm{at} 30^{\circ} \mathrm{C}$ for 30 minutes. Cells were observed under a phase-contrast microscope (top) and a fluorescence microscope (bottom). 
condition [21]. We thus examined the relationship between the fungicidal activity of $\mathrm{NM}$ and that arising from the combination of MC12 and AmB in terms of their effects on vacuole function and morphology. The yeast vacuoles were visible as a set of spherical membrane-enclosed compartments in untreated cells (Fig. 6a). They were observed as enlarged prominent architectures in cells treated with either $\mathrm{MC} 12$ or $\mathrm{AmB}$ alone at the nonlethal concentration (Fig. 6b,c). The AmB-induced morphological change of vacuoles seemed to reflect their fusion or expansion to a larger spherical structure, which is possibly effective for maintaining the cytoplasmic osmolarity balance. MC12 should promote vacuolar membrane fusion or expansion via a mechanism other than cellular osmoregulation, because MC12 did not cause plasma membrane permeability change at any of the concentrations tested. Vacuoles were not clearly visible in cells treated simultaneously with $\mathrm{MC} 12$ and $\mathrm{AmB}$ each at the nonlethal concentration, in which a fluorescence from FM4-64 was observed throughout the cytoplasm (Fig. 6d). NM similarly caused vacuolar membrane disability at its lethal concentration (Fig. 6e) as cytoplasmic distribution of the dye suggested leakage from the vacuolar membrane. In contrast, AmB-treated cells were characterized by intact vacuoles without the leakage of FM4-64 in medium even when the concentration of the antibiotic was increased up to $10 \mu \mathrm{M}$ (Fig. 6f). These findings support the idea that the combined actions of MC12 and AmB resulted in the generation of NM-like inhibitory activity on the machinery involved in vacuole morphogenesis or the maintenance of vacuolar membrane integrity.

\section{Transmission Electron Microscopy of Cells Treated with MC12, AmB, and Their Combination}

We finally observed cells under a transmission electron microscope to precisely analyze the effects of $\mathrm{MC12}$, AmB and their combination on the morphologies of vacuoles and other subcellular architectures. Vacuoles were visible as various membrane-enclosed compartments together with a nucleus in untreated cells (Fig. 7a), whereas MC12-treated cells were characterized by the appearance of an enlarged prominent vacuole (Fig. 7b). The vacuole was visible as highly expanded spherical architecture in AmB-treated cells (Fig. 7c). These transmission electron microscopy observations agreed with the fluorescence microscopy observations (Fig. 6). The most surprising feature of the combined actions of $\mathrm{MC} 12$ and $\mathrm{AmB}$ was the appearance of many vesicle-like compartments in addition to small vacuoles throughout the cytoplasm (Fig. 7d). The failure in clearly visualizing vacuoles with FM4-64 may be due to such an aberrant vacuole morphogenesis. Vacuole protein

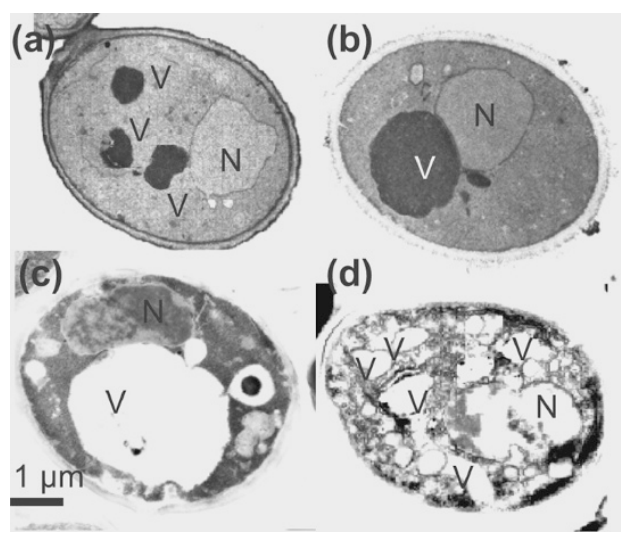

Fig. 7 Transmission electron microscopy of Saccharomyces cerevisiae cells incubated in YPD medium alone (a), medium containing $50 \mu \mathrm{M}$ MC12 (b), medium containing $1 \mu \mathrm{M} \mathrm{AmB} \mathrm{(c),} \mathrm{and} \mathrm{medium} \mathrm{containing} \mathrm{both}$ $50 \mu \mathrm{M} \mathrm{MC12}$ and $1 \mu \mathrm{M} \mathrm{AmB}$ (d) for 60 minutes at $30^{\circ} \mathrm{C}$.

$\mathrm{N}$ and $\mathrm{V}$ indicate nucleus-like and vacuole or vacuole-like architectures, respectively.

sorting (vps) mutants of $S$. cerevisiae exhibit defects in the sorting and processing of multiple vacuolar hydrolases, and are assigned to five distinct classes depending on vacuolar morphology and dynamics [10, 22]. Class C mutants lack any organelles resembling normal vacuoles, but accumulate vesicle-like aberrant architectures and are also characterized by the failure in their osmotic adaptation. The vesicle-like organelles seemed to be formed in cells treated with $\mathrm{MC} 12$ and $\mathrm{AmB}$ may result due to an impairment of the mechanism regulating vesicle assembly into vacuoles, as in the case of these mutants with the corresponding genetic defects, or in the corresponding cellular functions [23 25]. Unlike the case with these mutants, however, the membranous architectures of vacuoles and vesicle-like organelles were disrupted a least in part as judged from the image representing the leakage of FM4-64 (Fig. 6d). Nucleus-like organelles were also visible with abnormal morphology together with a disruptive damage in cells treated with $\mathrm{MC} 12$ and $\mathrm{AmB}$. These findings indicated the occurrence of severe fatal damage widely over the plasma membrane and intracellular membranous architectures as a result of the combined actions of MC12 and AmB.

This study reveals that the synergistic fungicidal activities of MC12 and AmB depend on the combination of various lethal events corresponding to those induced by NM including plasma membrane permeability change or plasma membrane disruption, ROS production, and aberrant vacuole morphogenesis. These cytotoxic events are not actually involved in the fungicidal activity of AmB when fungal cells are grown or incubated in nutrient 
medium under normal osmotic condition. Therefore, it is highly likely that the alkyl guanidinium chain of NM plays a major role in the fungicidal activity of NM in cooperation with the polyol lactone ring as its enhancer. Apoptosis-like events are induced in $S$. cerevisiae cells by the depletion of glutathione or by a low external dose of $\mathrm{H}_{2} \mathrm{O}_{2}$ [26]. Although ROS production is not necessarily a cause of cell death induction in $S$. cerevisiae, the fungicidal activity of $\mathrm{NM}$ could be attributed at least in part to its promotive effect on cellular ROS production via glutathione depletion $[4,8]$. Vacuoles contain several hydrolytic enzymes so that their leakage into the cytoplasm can be a cause of cell death progression [27]. The combined actions of $\mathrm{MC} 12$ and $\mathrm{AmB}$ on vacuoles may be a primary cause of inducing various downstream lethal events including ROS production. On the basis of the results of this study, we also propose that $\mathrm{AmB}$ can be a probe for developing vacuole-targeting antifungal agents.

\section{References}

1. Berlinck RG. Natural guanidine derivatives. Nat Prod Rep 19: 617-649 (2002)

2. Blinov NO, Golovkina LM, Khokhlova IM, Khlebarova EI, Kleiner EM, Georgieva IK, Sheikova GN, Koreniako AI. Study of the antibiotics of the endomycin group. Antibotiki 12: 867-874 (1967)

3. Grabley S, Hammann P, Raether W, Wink J, Zeeck A. Secondary metabolites by chemical screening. II. Amycins $\mathrm{A}$ and $\mathrm{B}$ two novel niphimycin analogs isolated from a high producer strain of elaiophylin and nigericin. J Antibiot 43: 639-647 (1990)

4. Nakayama K, Yamaguchi T, Doi T, Usuki Y, Taniguchi M, Tanaka T. Synergistic combination of direct plasma membrane damage and oxidative stress as a cause of antifungal activity of polyol macrolide niphimycin. J Biosci Bioeng 94: 207-211 (2002)

5. Usuki Y, Matsumoto K, Inoue T, Yoshioka K, Iio H, Tanaka T. Structure-activity relationship studies on niphimycin, a guanidylpolyol macrolide antibiotic. Part 1: The role of the $N$-methyl- $N$-alkylguanidinium moiety. Bioorg Med Chem Lett 16: 1553-1556 (2006)

6. Tanaka T, Nakayama K, Machida K, Taniguchi M. Longchain alkyl ester of AMP acts as an antagonist of glucoseinduced signal transduction that mediates activation of plasma membrane proton pump in Saccharomyces cerevisiae. Microbiology 146: 377-384 (2000)

7. Davis LE, Shen J, Royer RE. In vitro synergism of concentrated Allium sativum extract and amphotericin B against Cryptococcus neoformans. Planta Med 60: 546-549 (1994)

8. Ogita A, Hirooka K, Yamamoto Y, Tsutsui N, Fujita K,
Taniguchi M, Tanaka T. Synergistic fungicidal activity of $\mathrm{Cu}^{2+}$ and allicin, an allyl sulfur compound from garlic, and its relation to the role of alkylhydroperoxide reductase 1 as a cell surface defense in Saccharomyces cerevisiae. Toxicology 15: 205-213 (2005)

9. Machida K, Tanaka T, Fujita K, Taniguchi M. Farnesolinduced generation of reactive oxygen species via indirect inhibition of mitochondrial electron transport chain in Saccharomyces cerevisiae. J Bacteriol 180: 4460-4465 (1998)

10. Vida TA, Emr SD. A new vital stain for visualizing vacuolar membrane dynamics and endocytosis in yeast. J Cell Biol 128: 779-792 (1995)

11. Ghannoum MA, Rice LB. Antifungal agents: mode of action, mechanisms of resistance, and correlation of these mechanisms with bacterial resistance. Clin Microbiol Rev 12: 501-519 (1999)

12. Baginski M, Sternal K, Czub J, Browski E. Molecular modelling of membrane activity of amphotericin B, a polyene macrolide antifungal antibiotic. Acta Biochim Pol 52: 655-658 (2005)

13. Brajtburg J, Elberg S, Schwartz DR, Vertut-Croquin A, Schlessinger D, Kobayashi GS, Medoff G. Involvement of oxidative damage in erythrocyte lysis induced by amphotericin B. Antimicrob Agents Chemter 27: 172-176 (1985)

14. Sokol-Anderson M, Sligh Jr JE, Elverg S, Brajtburg J, Kobayashi GS, Medoff G. Role of cell defense against oxidative damage in the resistance of Candida albicans to the killing effect of amphotericin B. Antimicrob Agents Chemter 32: 702-705 (1988)

15. Okamoto Y, Aoki S, Mataga I. Enhancement of amphotericin B activity against Candida albicans by superoxide radical. Micropathol 158: 9-15 (2004)

16. Andrews FA, Beggos WH, Sarosi GA. Influence of antioxidants on the bioactivity of amphotericin B. Antimicrob Agents Chemter 11: 615-618 (1977)

17. Beggs WH, Andrews FA, Sarosi GA. Synergistic action of amphotericin B and antioxidants against certain opportunistic yeast pathogens. Antimicrob Agents Chemter 13: 266-270 (1978)

18. Osaka K, Ritov VB, Bernardo JF, Branch RA, Kagan VE. Amphotericin B protects cis-parinaric acid against peroxyl radical-induced oxidation: amphotericin $\mathrm{B}$ as an antioxidant. Antimicrob Agents Chemter 41: 743-747 (1997)

19. Efe JA, Botelho RJ, Emr SD. The Fab1 phosphatidylinositol kinase pathway in the regulation of vacuole morphology. Curr Opin Cell Biol 17: 402-408 (2005)

20. Bhuiyan MS, Ito Y, Nakamura A, Tanaka N, Fujita K, Fukui $\mathrm{H}$, Takegawa K. Nystatin effects on vacuolar function in Saccharomyces cerevisiae. Biosci Biotechnol Biochem 63: 1075-1082 (1999)

21. Ogita A, Fujita K, Taniguchi M, Tanaka T. Enhancement of the fungicidal activity of amphotericin B by allicin, an allylsulfur compound from garlic, against the yeast 
Saccharomyces cerevisiae as a model system. Planta Med 72: 1247-1250 (2006)

22. Banta LM, Robinson JS, Klionsky DJ, Emr SD. Organelle assembly in yeast: characterization of yeast mutant defective in vacuolar biogenesis and protein sorting. J Cell Biol 107: 1369-1383 (1988)

23. Rieder SE, Emr SD. A novel RING finger protein complex essential for a late step in protein transport to the yeast vacuole. Mol Biol Cell 8: 2307-2327 (1997)

24. Sato TK, Rehling P, Peterson MR, Emr SD. Class C Vps protein complex regulates vacuolar SNARE pairing and is required for vesicle docking/fusion. Mol Cell 6: 661-671
(2000)

25. Srivastava A, Woolford CA, Jones EW. Pep3p/Pep5p complex: a putative docking factor at multiple steps of vesicular transport to the vacuole of Saccharomyces cerevisiae. Genetics 156: 105-122 (2000)

26. Madeo F, Frohilich E, Liger M, Grey M, Sigrist SJ, Wolf $\mathrm{DH}$, Frohlich KU. Oxygen stress: a regulator of apoptosis in yeast. J Cell Biol 145: 757-767 (1999)

27. Wiemken A, Schellenberg M, Urech K. Vacuoles: the sole compartments of digestive enzyme in yeast. Arch Microbiol 123: 23-35 (1979) 